\title{
Etude de l'écoulement moyen en sortie de roue d'une pompe centrifuge (Roue SHF)
}

\author{
Flow study at the exit of a centrifugal pump impeller \\ par M. El Hajem*, R. Morel*, F. Spettel*, G. Bois** \\ * Equipe Hydraulique I.N.S.A Lyon \\ ** Ingénieur Metraflu \\ Laboratoire de Mécanique des Fluides et d'Acoustique, E.C.L, UMR CNRS 5509
}

Flows in centrifugal pumps are one of the most complex encountered in turbomachinery. The flow leaving the impeller is affected by the presence of downstream elements such as the diffuser or the volute. This paper presents the results of flow investigation at the exit of a centrifugal impeller run with a vanned diffuser and a spiral casing of industrial type. Velocity and pressure measurements with a directional probe were obtained at three sections located at different circumferential distances from the volute tongue. For each of these sections, traverses were made at two distances from the diffuser vane leading edge. The results showed an asymmetric flow field around the rotor depending mainly on the diffuser arrangement, while the effect of the volute is less pronounced. With the impeller running at partial load, this dependency is influenced by additional phenomena such as back flows and more important gap flows.

\section{I 口INTRODUCTION}

En dehors des conditions de dessin d'une machine centrifuge, l'environnement aval de la roue influe sur le fonctionnement de celle-ci. La présence du diffuseur entraîne une distorsion non négligeable de l'écoulement en sortie de roue. L'écoulement devient plus complexe lorsque le diffuseur est aubé du fait de l'interaction des deux rangées d'aubes. Pour éliminer l'influence de la présence d'une volute, les études, en air ou en eau, sont souvent menées avec des volutes de grandes dimensions ou même en l'absence de volute [8], [10]. Dans le cas contraire, l'analyse du rôle joué par la volute est souvent faite en l'absence de diffuseur [4], [7], [9]. Pour l'étude actuelle, nous nous sommes intéressés au cas où les deux organes sont présents. Le but recherché est d'arriver à dissocier l'influence du diffuseur de celle de la volute afin d'évaluer leurs effets respectifs.

La roue étudiée est un modèle en eau de la roue SHF qui a été conçue à l'origine pour un programme de recherche portant sur l'étude de la recirculation [2], [8]. Par la suite, d'autres études ont été essentiellement consacrées à l'analyse de l'écoulement interne dans les deux modèles existants : le modèle en eau [3], et le modèle en air [10].

Cet article décrit l'exploration du champ hydraulique en sortie de roue. Les résultats présentés sont issus des mesures effectuées à la sonde directionnelle en quatre points de fonctionnement allant de $\mathrm{Q}^{*}=1$ à $\mathrm{Q}^{*}=0,5$ et en différentes positions azimutales par rapport au diffuseur et à la volute.

\section{II — MACHINE D'ESSAIS}

La roue de diamètre extérieur de $0,354 \mathrm{~m}$, comprend 7 aubes à double courbure. Elle a été dessinée pour les caractéristiques adimensionnelles suivantes :

— coefficient de débit : $\quad \phi=Q_{n} /\left(2 \pi R_{2}^{2} b \omega\right)=0,118$

- coefficient de hauteur : $\psi=g H_{n} /\left(\omega^{2} R_{2}^{2}\right)=0,481$

- rapidité spécifique : $\quad \omega_{s n}=\omega Q_{n}^{1 / 2} /\left(g H_{n}\right)^{3 / 4}=0,577$

Les essais se sont déroulés sur la boucle d'essais de pompe centrifuge du LMF (fig. 1). La roue est suivie d'un 


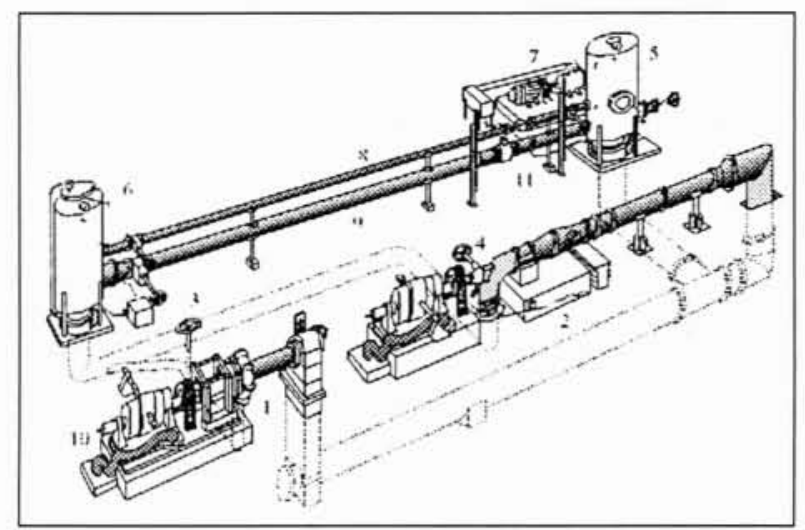

1. Boucle d'essais.

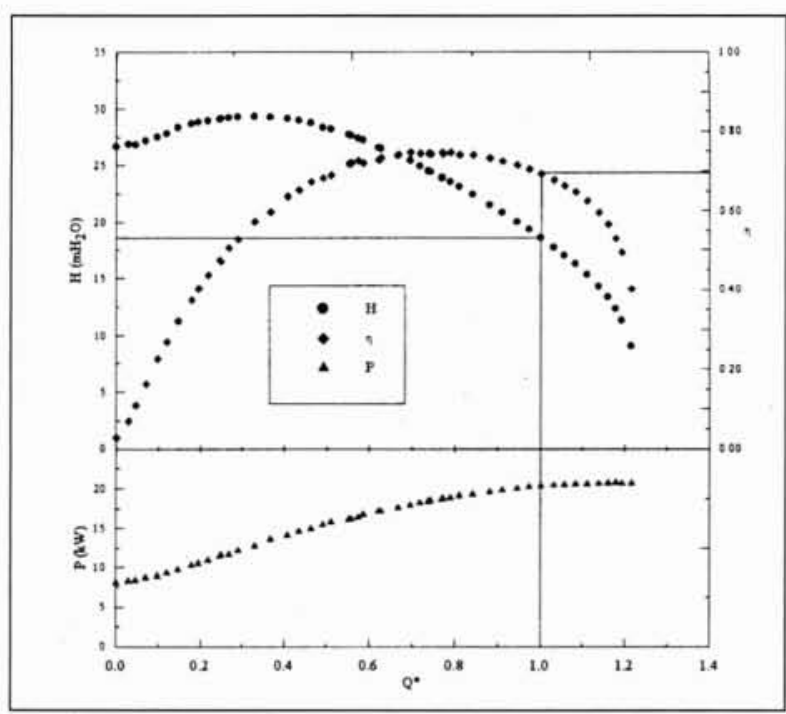

\section{Caractéristiques de la machine à $1200 \mathrm{tr} / \mathrm{mn}$.}

diffuseur à parois parallèles dont le flasque arrière, sur lequel sont montées 6 aubes, est libre en rotation. Ainsi, il est possible d'effectuer, pour une même position de la volute, plusieurs explorations azimutales par rapport au diffuseur. Le premier sondage correspond à un plan pour lequel

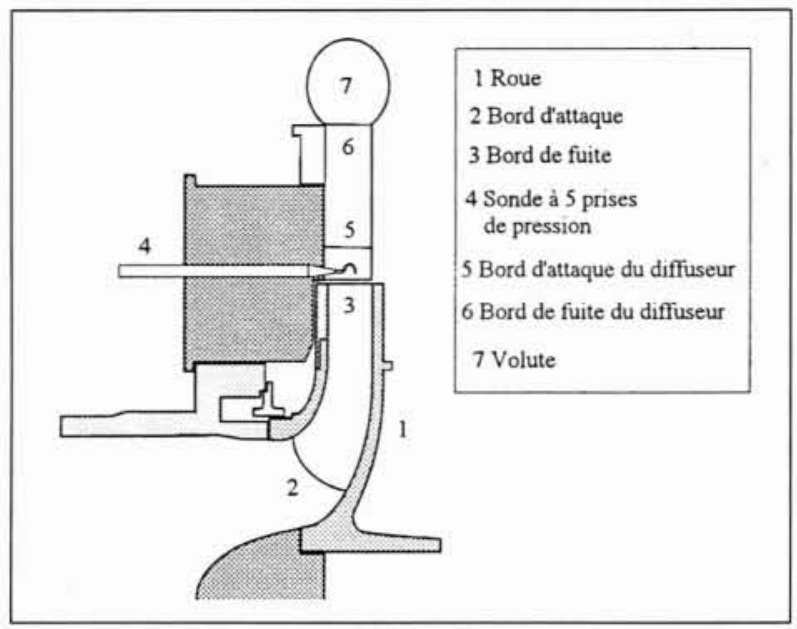

3. Vue méridienne des sections de mesure.

l'écoulement a été également exploré au moyen d'un anémomètre laser à effet Doppler ; cela nous a permis de confronter les résultats obtenus par les deux techniques [5].

De même, les mesures effectuées par le biais de la sonde donnent accès aux caractéristiques globales en sortie de la roue : débits, hauteur et puissance. Ces dernières sont également comparées avec les grandeurs globales de la machine (fig. 2). Ces différentes comparaisons ont abouti à des résultats satisfaisants [5].

Les sondages sont effectués sur un rayon situé à $8 \mathrm{~mm}$ en aval du bord de fuite (fig. 3). Deux autres positions azimutales relatives à la volute ont été explorées. Elles sont repérées par l'angle $\theta$ dont l'origine correspond au bec de volute (fig. 4). L'ensemble du dispositif de mesures et de traitement des données est décrit dans [5].

\section{III —CHAMP HYDRAULIQUE}

Les essais ont couvert une plage de fonctionnement allant de $Q^{*}=1,1$ à 0,5 , on se limitera dans ce papier à présenter les résultats obtenus à $Q^{*}=1 ; 0,8$ et 0,5 . Nous nous sommes intéressés à l'étude des champs moyens de vitesse et de pression. Les composantes de la vitesse ont été adimensionnées par la vitesse d'entraînement $U_{2}$ et sont présentées en

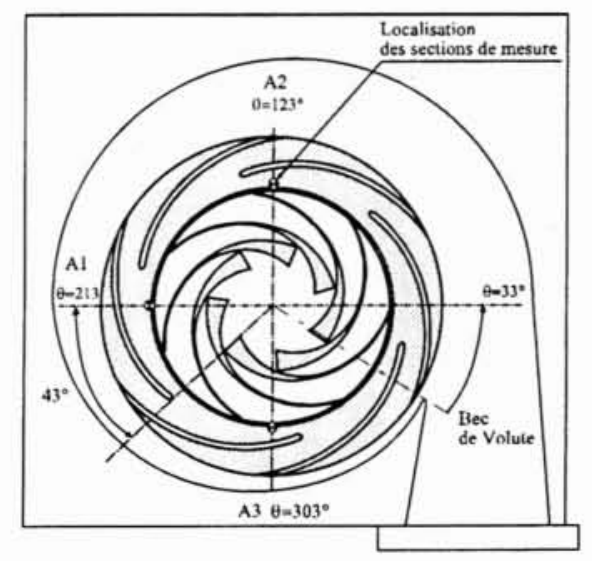

$-a-$

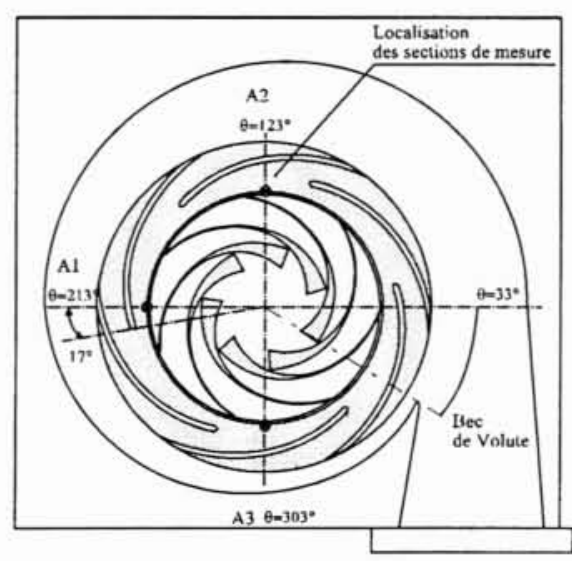

$-b-$

4. Plans de sondage. 


\section{Applications industrielles de la mécanique des fluides}

fonction de la distance axiale réduite $z / b, b$ étant la largeur en sortie de roue. Les pressions statique et totale sont mesurées par rapport à une pression de référence mesurée dans une section amont non touchée par la prérotation.

\subsection{Champ de vitesse}

\subsubsection{Vitesse radiale}

Les figures 5 représentent le profil de la composante radiale de la vitesse respectivement aux débits $Q^{*}=1 ; 0,8$ et 0,5 . Il nous a paru naturel de présenter les résultats en deux ensembles correspondant chacun à une même position par rapport au diffuseur. Un premier groupe se compose des mesures issues des sondages en $A_{1} D_{1}, A_{2} D_{2}$ et $A_{3} D_{2}$, un deuxième correspond aux sondages en $A_{1} D_{2}, A_{2} D_{1}$ et $A_{3} D_{1}$.

Au débit nominal (fig. $5 a$ ), on constate que les vitesses constituant le deuxième groupe sont plus faibles que celles du premier groupe. Cela correspond à un blocage dû à la proximité des bords d'attaque des aubes du diffuseur. La dispersion des valeurs de $C_{r}$ pour un groupe donné est due à la présence de la volute. Cet effet semble être de moindre importance à proximité du bord d'attaque du diffuseur (groupe 2). A ce stade, on peut considérer que la présence du diffuseur apporte des distorsions d'amplitude plus grande que celle provenant de la volute.

La vitesse radiale évolue significativement avec le débit. A $0,8 Q_{n}$, l'ensemble des résultats indique un écoulement uniforme autour de la roue (fig. $5 b$ ). On peut ainsi considérer que l'adaptation roue-diffuseur est obtenue à $0,8 Q_{n}$. Il ne reste, a priori, que les distorsions liées à la présence de la volute.

Pour un débit plus faible $\left(Q^{*}=0,5\right)$, l'écoulement redevient asymétrique (fig. $5 c$ ). Toutefois, la vitesse radiale est maintenant plus importante à $\psi=17$ degrés avec un écart de $30 \%$ en $A_{3}$ et d'environ $22 \%$ en $A_{1}$ et $A_{2}$.

L'analyse locale de la vitesse radiale à proximité du plafond et de la ceinture fait apparaitre des zones de recirculation à partir de $0,7 Q_{n}$ en $A_{1} D_{2}$. A $0,6 Q_{n}$, la recirculation s'étend d'avantage du côté de la ceinture à l'exception de $A_{3}$. En $A_{1}$ ainsi que pour $A_{2} D_{1}$, elle occupe $17 \%$ de la largeur de la roue. En $A_{2} D_{2}$, l'écoulement de retour occupe $9 \%$ du passage. Pour cette même section ainsi que pour $A_{3} D_{2}$, une recirculation commence à apparaître au voisinage du plafond [5]. A $0,5 Q_{n}$, l'écoulement de recirculation est présent à proximité des deux flasques.

L'apparition de la recirculation vers le plafond est accompagnée d'une réduction de l'écoulement de retour au voisinage de la ceinture. Dans cette région, elle n'occupe plus que $10 \%$ du passage en $A_{1}$ et $15 \%$ pour $A_{2}$. Par ailleurs, la recirculation en $A_{3}$ est plus importante, au voisinage du plafond où elle s'étend sur $12 \%$ de la largeur de la roue. En revanche, elle est absente du côté de la ceinture.

\subsubsection{Vitesse tangentielle}

Comme pour la vitesse radiale, les résultats montrent un effet lié à la présence du diffuseur (fig. 6). Néanmoins, ce
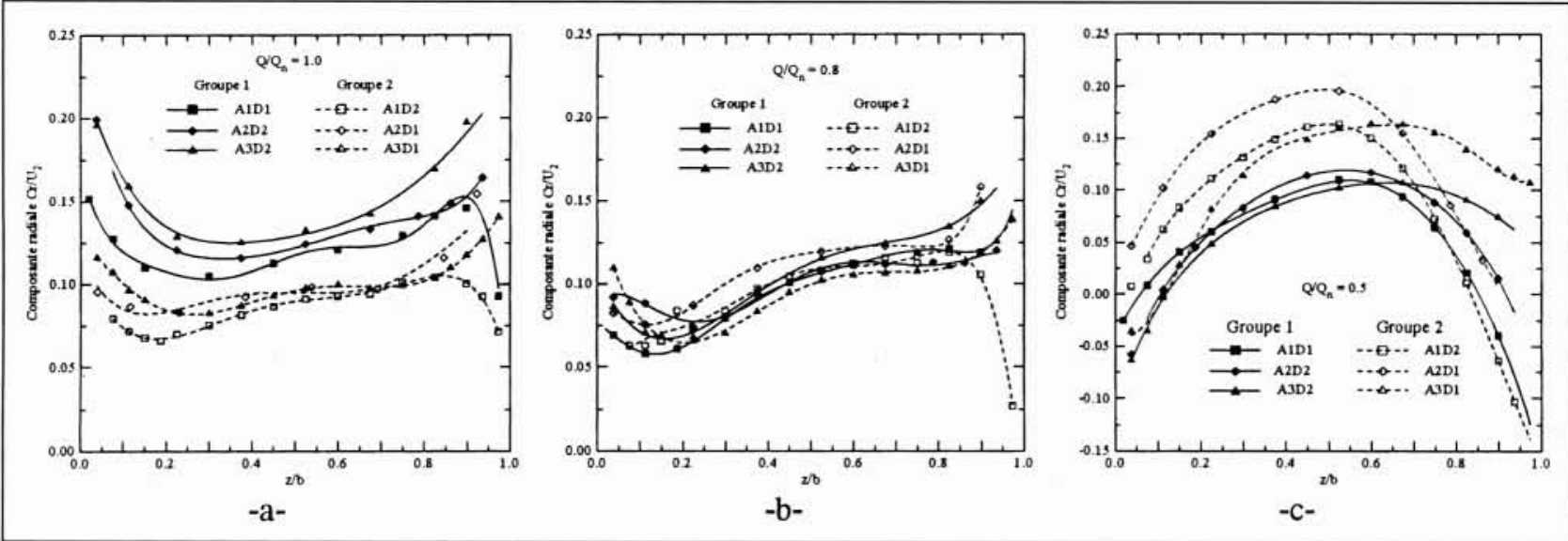

5. Vitesse radiale $C_{r} / U_{2}$, à $Q_{n}(\mathrm{a}), 0,8 Q_{n}(\mathrm{~b}), 0,5 Q_{n}(\mathrm{c})$.
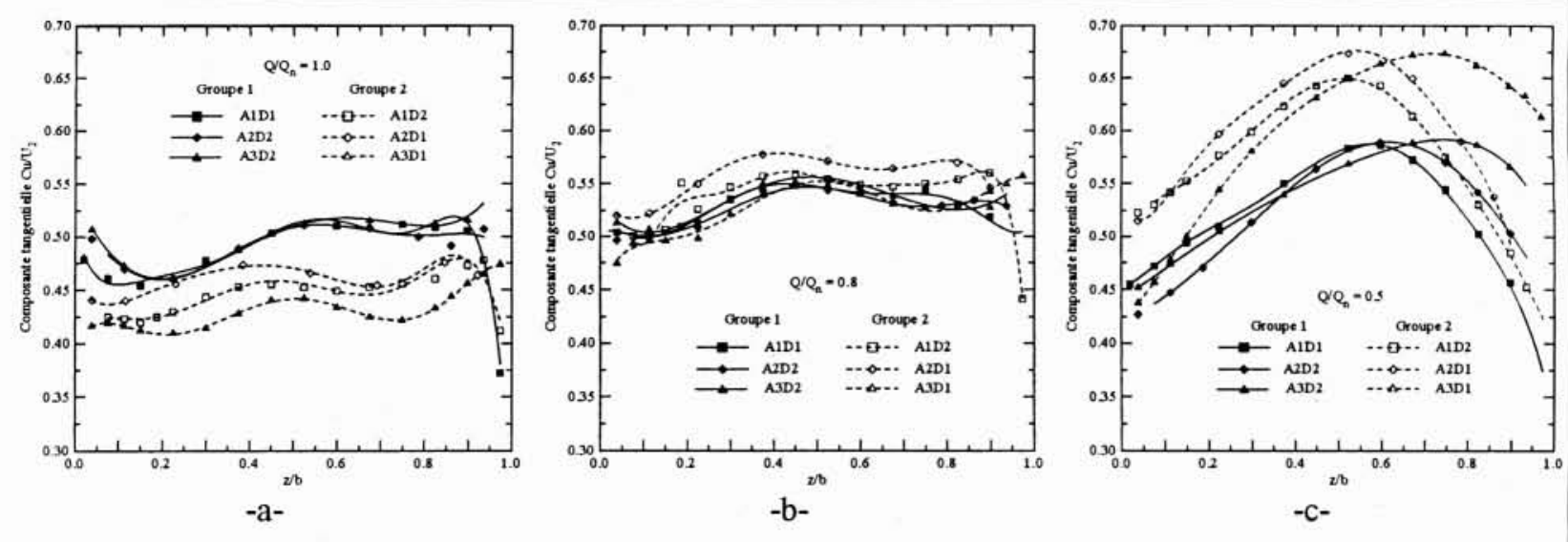

6. Vitesse tangentielle $C_{u} / U_{2}$ à $Q n(\mathrm{a}), 0,8 Q_{n}(\mathrm{~b}), 0,5 Q_{n}$ (c). 
sont les vitesses obtenues pour le groupe 1 , constitué des sections les plus éloignées des aubes du diffuseur, qui sont les plus homogènes. Nous pouvons en conclure que pour ce groupe, la vitesse tangentielle n'est pas influencée par la présence de la volute au débit nominal. Cela n'est pas le cas du groupe 2 pour lequel l'effet de la volute se manifeste davantage à proximité du bord d'attaque des aubes du diffuseur, zone où la composante radiale $\mathrm{Cr}$ devient plus faible. A $0,8 Q_{n}$, l'écoulement est plus homogène, l'écart entre les deux groupes est moins important. A plus faible débit (fig. 6c), on constate que les vitesses du groupe 2 sont plus importantes que celles du premier contrairement à la distribution observée à plus grands débits.

\subsubsection{Vitesse axiale}

La figure 7 montre une distribution homogène de la composante axiale de la vitesse dans l'entrefer roue-diffuseur. L'écoulement est globalement orienté en direction de la ceinture et reste quasiment inchangé quand le débit varie. Seules les mesures obtenues en A3D1 montrent un comportement différent de l'écoulement qui est orienté en direction du plafond. L'important gradient de vitesse présent à proximité de la ceinture est à attribuer à l'écoulement de jeu existant entre la sortie de la roue et l'ouïe d'aspiration, cet écoulement étant dû à la différence de pression entre la sortie et l'entrée de la roue.

\subsubsection{Angle de l'écoulement relatif}

L'asymétrie de l'écoulement se retrouve aussi dans la distribution de l'angle de l'écoulement relatif (fig. 8). Au voisinage des parois, l'angle de l'écoulement se rapproche de l'angle géométrique sous l'influence des écoulements secondaires qui conduisent à un faible glissement à proximité des parois [5]. Au débit d'adaptation roue-diffusuer $\left(Q^{*}=0,8\right)$, l'écoulement s'aligne sur la direction des aubes du diffuseur.

\section{- 3.2 Distribution du champ de pression}

Les données sont présentées sous formes de coefficients de pression définis comme suit :

- coefficient de pression statique :

$$
C_{p}=\frac{p_{2}-p_{1}}{\frac{1}{2} \rho U_{2}^{2}}
$$

- coefficient de pression totale :

$$
C_{p t}=\frac{p_{2}^{0}-p_{1}}{\frac{1}{2} \rho U_{2}^{2}}
$$

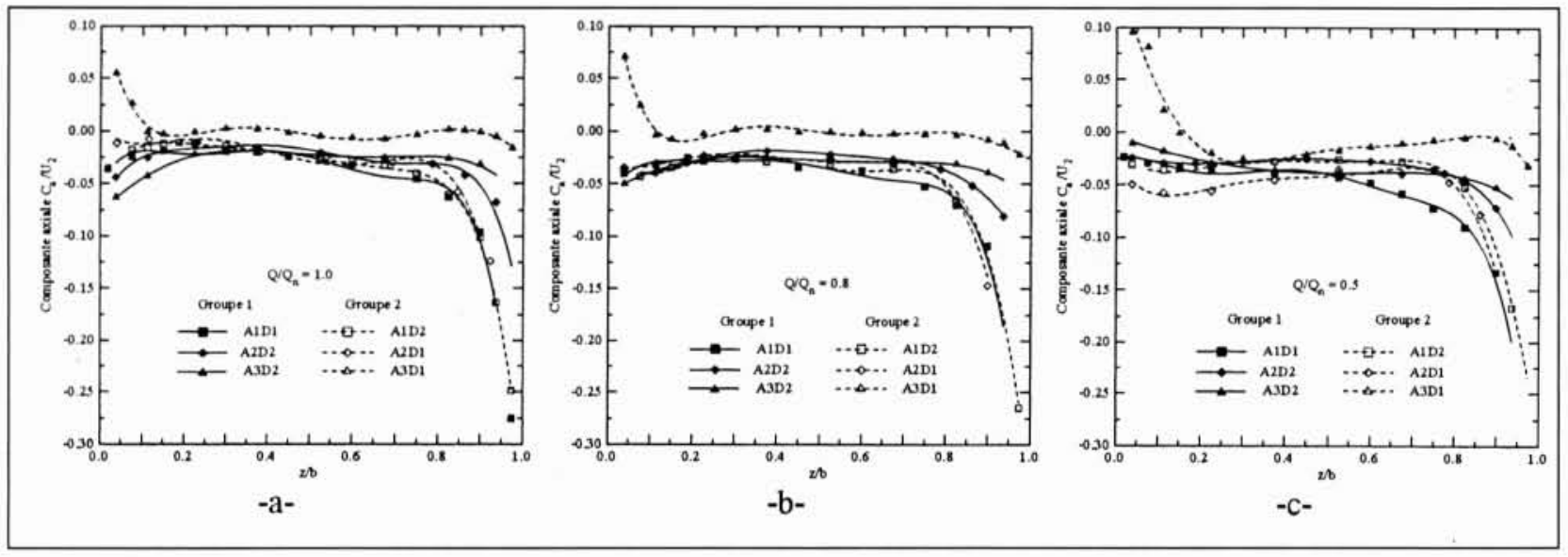

7. Vitesse axiale à $Q_{n}(\mathrm{a}), 0,8 Q_{n}(\mathrm{~b}), 0,5 Q_{n}$ (c).

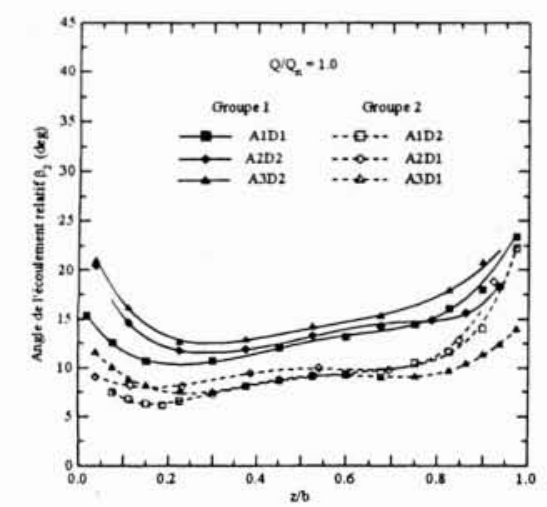

-a-

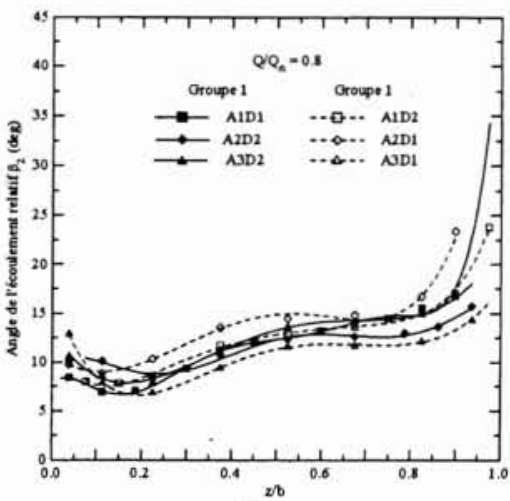

-b-

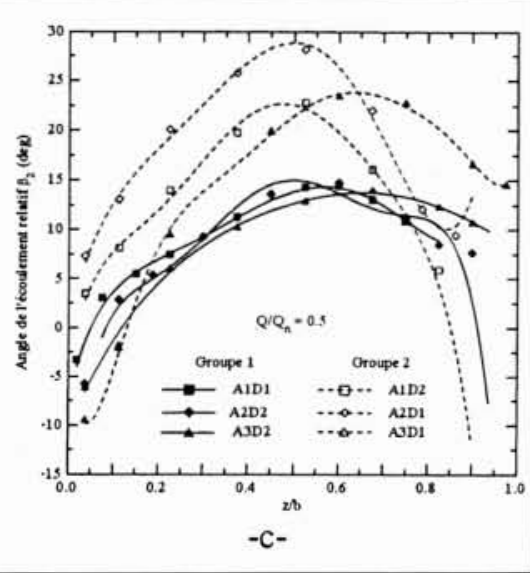

8. Distribution de $\beta_{2}$ à $Q_{n}$ (a), $0,8 Q_{n}$ (b), $0,5 Q_{n}$ (c). 


\subsubsection{Pression statique}

D'une façon générale, la pression statique varie peu le long de la ligne de mesure, ce qui tend à prouver que la forme des lignes de courant évolue peu sur la hauteur de l'aube, sauf éventuellement pour les dix derniers pour-cents côté ceinture. Autrement dit, la mesure des pressions pariétales sur les deux flasques peut être représentative du gradient de pression réel.

Par ailleurs, la présence du diffuseur semble être à l'origine d'un champ de pression statique non uniforme autour de la roue sauf au point d'adaptation $\left(Q^{*}=0,8\right)$. Au débit nominal (fig. 9a), la variation azimutale de la pression dépend essentiellement de la position par rapport au diffuseur. En milieu de veine, l'écart maximum entre $\psi_{1}$ et $\psi_{2}$ est de $9 \%$; il est enregistré en $A_{3}$. En revanche, le maximum d'écart n'est que de $3 \%$ entre les sections $A_{1}$ et $A_{3}$ pour $\psi=43^{\circ}$. A $0,8 Q_{n}$, la pression statique est plus homogène en sortie de roue. La variation de $C_{p}$ ne dépasse pas $5 \%$ de la valeur enregistrée pour la section de référence $A_{1} D_{1}$ (fig. $9 b$ ). Pour un débit plus faible, une importante modification du champ de pression est observée (fig. $9 c$ ) ; l'effet de la volute se manifeste davantage à proximité des bords d'attaque du diffuseur côté plafond.

A fort débit, l'écoulement est non adapté par rapport au bec de volute. Une fraction de l'écoulement ne pouvant être évacuée repasse à nouveau dans la volute à travers le jeu existant entre le bec de volute et le diffuseur. L'importante accélération de l'écoulement qui résulte de la réduction de la section de passage s'accompagne d'une chute de la pression statique [1], [4]. Cette chute de pression expliquerait alors les valeurs enregistrées pour la section $A_{3}$ la plus proche du bec de volute. A sous-débit, on a constaté que le champ de pression dans la volute évolue considérablement en fonction de l'azimut. Les variations dépendent de la forme et des conditions de fonctionnement de celle-ci et aucune tendance générale n'est observée [1], [4] [11]. Dans notre cas, des mesures complémentaires de pression statique dans la volute s'avèrent alors nécessaires pour une meilleure évaluation de son action sur l'écoulement en sortie de roue.

\subsubsection{Pression totale}

Les variations de la pression totale sont dues aux variations simultanées de la pression statique et de la vitesse tangentielle en sortie de roue. Le profil de la pression totale est comparable à celui de la vitesse tangentielle. La pression statique relativement constante entre les flasques n'introduit pas de distorsion significative de la pression totale dans la direction axiale (fig. 10). A proximité du plafond, la pression totale est uniforme autour de la roue, ce qui n'est pas le cas au voisinage de la ceinture. Dans cette région, la pression totale est plus importante pour le premier groupe, donc pour les sections les plus éloignées des aubes du diffuseur. En ce qui concerne l'action de la volute, elle semble être plus marquée pour le groupe 2 c'est-à-dire pour les sections situées le plus proche des aubes du diffuseur.

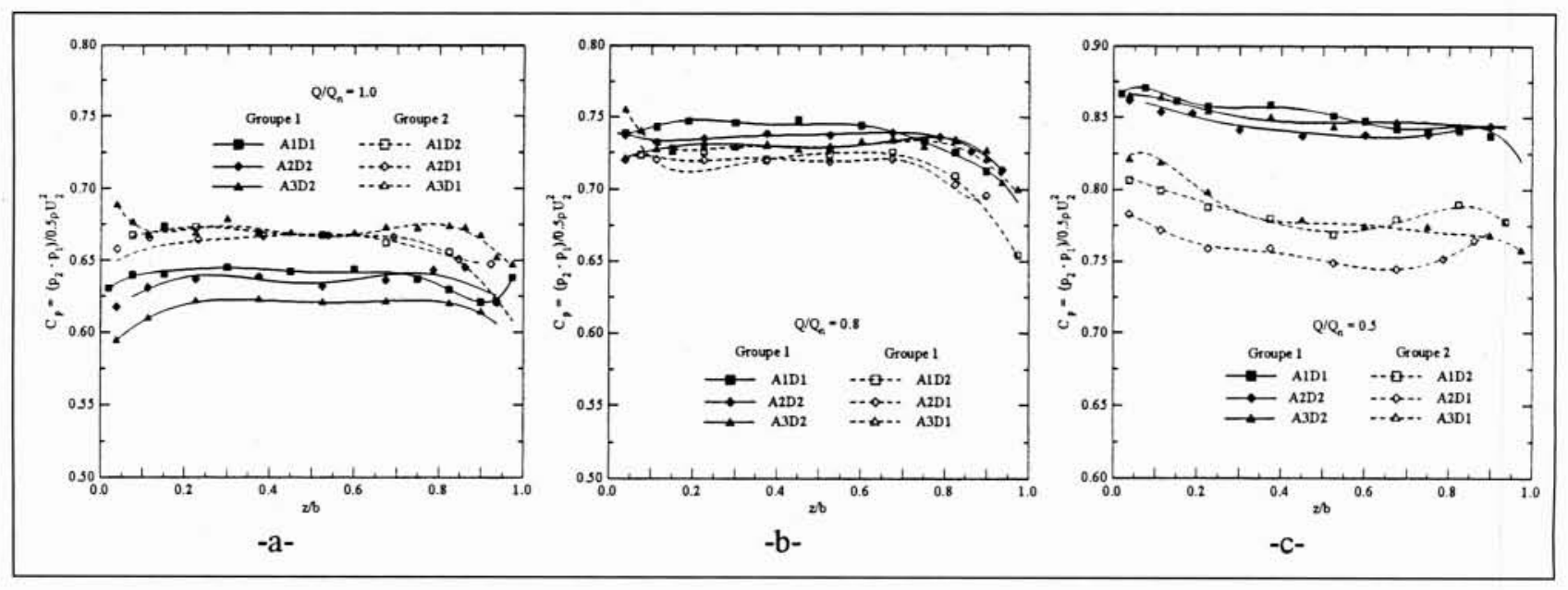

9. Coefficient de pression statique à $Q_{n}(\mathrm{a}), 0,8 Q_{n}(\mathrm{~b}), 0,5 Q_{n}(\mathrm{c})$.

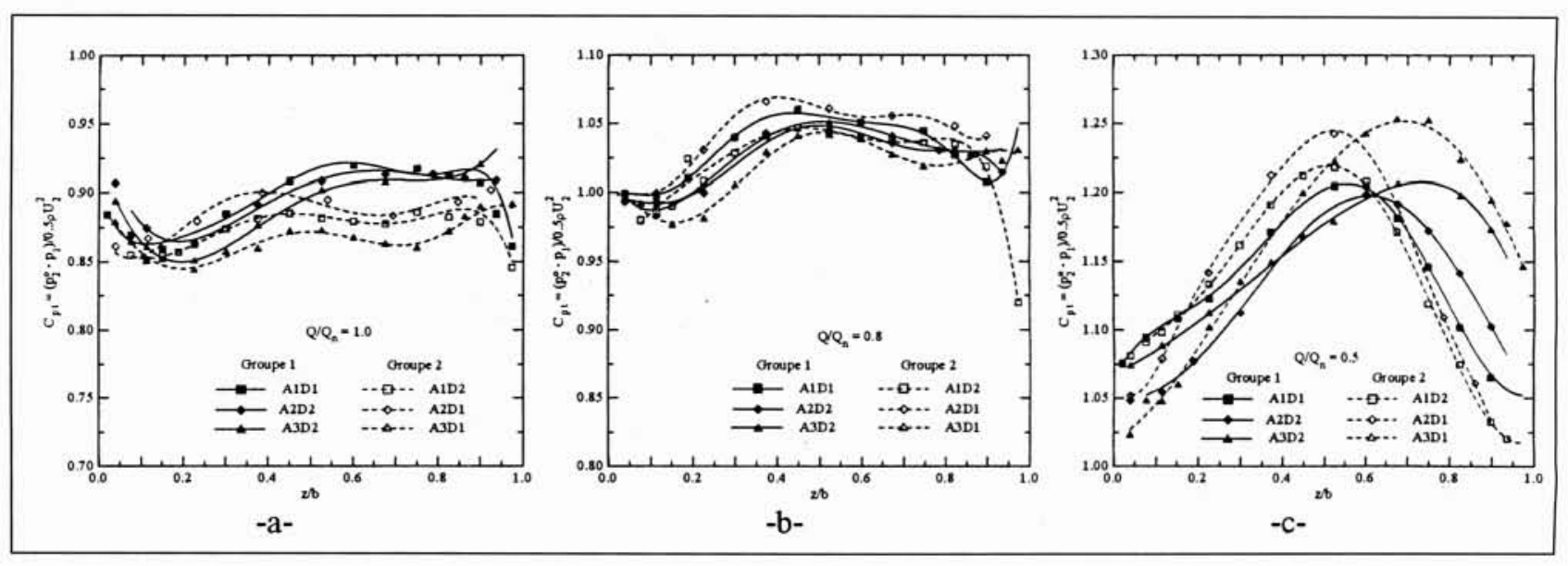

10. Coefficient de pression totale à $Q_{n}(\mathrm{a}), 0,8 Q_{n}(\mathrm{~b}), 0,5 Q_{n}$ (c). 


\section{ANALYSE DU GLISSEMENT EN SORTIE DE ROUE}

Pour le coefficient de glissement qui caractérise la déviation [5], on se limitera à l'analyse des résultats relatifs aux sections $A_{1} D_{1}$ et $A_{1} D_{2}$ (fig. ll). L'évolution de ce coefficient montre un net écart entre le premier et le deuxième groupe de mesures.

Pour le premier groupe et au milieu de la veine, le glissement est uniforme et n'est affecté que faiblement par la variation du débit. Ce résultat peut s'expliquer par le fait que la variation de $C_{r}$ est compensée par un changement de la direction de l'écoulement, donc de $\beta_{2}$, de façon à maintenir constante la vitesse de glissement. La valeur moyenne du coefficient de glissement est d'environ 0,8 ; elle correspond à une faible déviation dans la roue. Au voisinage des flasques et à très faible débit, l'important glissement peut s'expliquer par l'apparition de la recirculation.

Pour le deuxième groupe, on peut noter une importante variation du coefficient de glissement d'un sondage à l'autre qui s'explique par la présence des aubes du diffuseur, à l'exception de $Q^{*}=0,8 Q_{n}$, débit pour lequel l'écoulement est uniforme en sortie de roue. Pour ce débit le glissement est alors du même ordre de grandeur que celui enregistré pour le premier groupe.

Enfin, nous avons reporté dans le tableau 1 les résultats d'une comparaison entre la valeur moyenne à mi-hauteur $(\xi=0,8)$ et le coefficient de glissement obtenu par différentes formules empiriques existant dans la littérature [5]. A noter le plus faible écart obtenu pour les corrélations de Fujié et Stodola.

Tableau 1. - Coefficient de glissement calculé par différentes corrélations.

\begin{tabular}{|l|c|c|}
\hline Modèle & $\begin{array}{c}\text { Coefficient } \\
\text { de glissement }\end{array}$ & Ecart (\%) \\
\hline Balje & 0,65 & 18 \\
\hline Fujié & 0,789 & 1,4 \\
\hline Pfleiderer & 0,855 & 6,9 \\
\hline Stanitz & 0,717 & 10 \\
\hline Stodola & 0,828 & 3,5 \\
\hline Wiesner & 0,841 & 5,1 \\
\hline
\end{tabular}

\section{V a ANALYSE DES PERFORMANCES DE LA ROUE}

\subsection{Distribution du débit en sortie de roue}

Outre l'évolution du champ de vitesse et de pression, la répartition du débit à travers la largeur de la roue donne une meilleure indication quant au fonctionnement de celle-ci. Ainsi, chaque section de mesure a été partagée en deux régions. La première zone s'étend de la ceinture au milieu du canal ; la deuxième s'étend du milieu du canal jusqu'au plafond. Chacune de ces deux régions est traversée par un débit calculé par intégration de la composante débitante de la vitesse :

— côté plafond:

$$
\bar{C}_{r}=\frac{1}{\frac{1}{2} b} \int_{0}^{b / 2} C_{r} d z
$$

- côté ceinture :

$$
\bar{C}_{r}=\frac{1}{\frac{1}{2} b} \int_{b / 2}^{b} C_{r} d z
$$

le débit local est alors donné par :

$$
Q_{l}=\frac{1}{2} b R_{2} \int_{0}^{2 \pi} \bar{C}_{r} d \theta
$$

Les résultats de ce calcul sont reportés sur la figure 12. Dans cette représentation, l'écoulement est uniformément réparti si tous les points sont alignés à 0,5 . On constate que la répartition du débit dépend à la fois de la section de mesure et du point de fonctionnement considéré. Cependant, on observe, avant l'apparition de la recirculation, une même répartition pour les positions $A_{1} D_{1}, A_{2} D_{2}$ et $A_{3} D_{2}$. Là encore, on peut en conclure une faible dépendance de l'écoulement en sortie de roue vis-à-vis de la volute pour les débits supérieurs à $0,67 Q_{n}$. Pour des débits plus faibles, on enregistre une véritable redistribution de l'écoulement autour de la roue. Suite à l'apparition de la recirculation vers la ceinture, un report de l'écoulement s'effectue au profit des filets situés à proximité du plafond. Mais en dessous de $0,5 Q_{n}$, la région du plafond est à son tour affectée par l'écoulement de retour, on constate alors un rééquilibrage du débit à travers la veine d'essais.

Par ailleurs, on observe qu'à $0,67 Q_{n}$, une importante modification de l'écoulement est présente. Elle implique une véritable redistribution de l'écoulement à travers des sections de mesures. Un report de l'écoulement s'effectue au profit
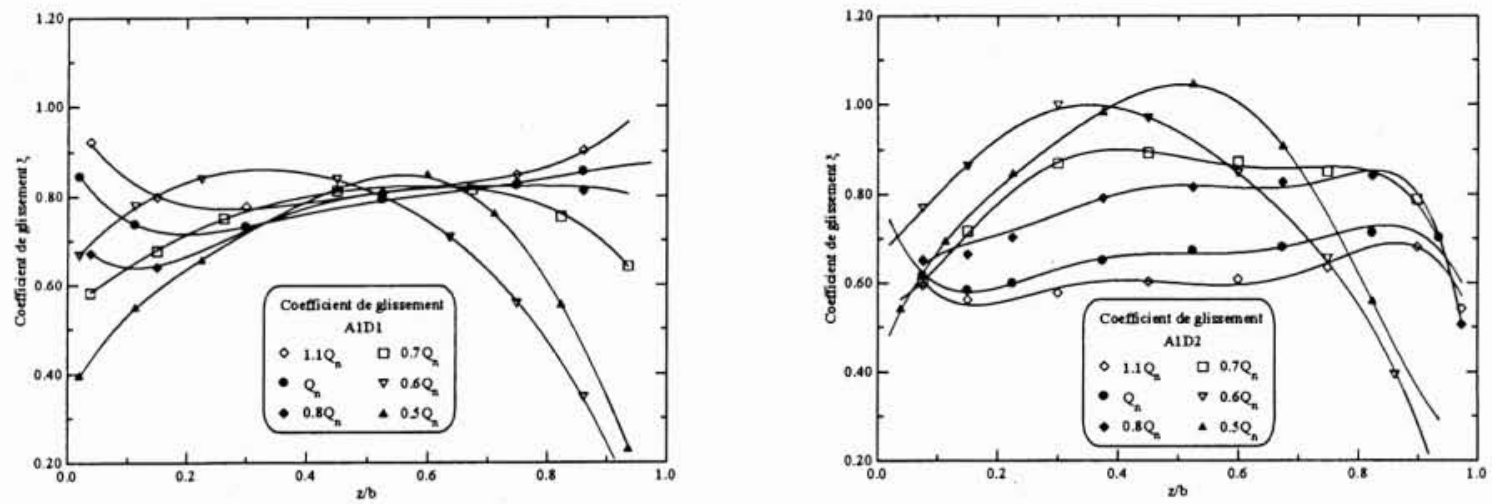

11. Coefficient de glissement. 


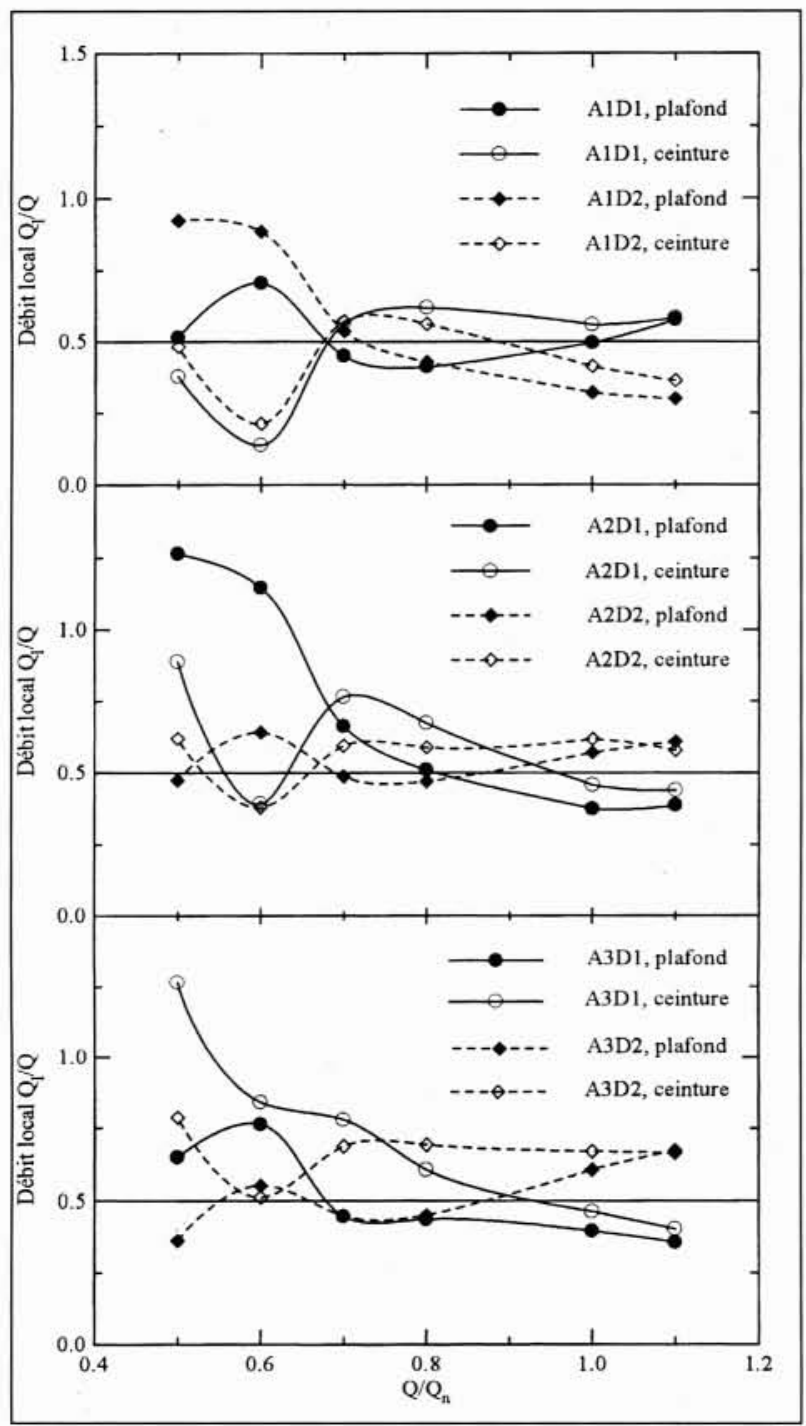

12. Distribution des débits locaux.

des filets situés à proximité du plafond. Cette redistribution des débits locaux est le résultat de l'apparition de la recirculation en premier lieu vers la ceinture. A $0,5 Q_{n}$, la région du plafond est à son tour occupée par la recirculation. On constate, de nouveau, un rééquilibrage du débit à travers la veine d'essais.

\subsection{Rendement de la roue}

Le rendement hydraulique est étudié localement pour des tubes d'isodébit que l'on peut assimiler à des tubes de courant. Pour chacun de ces tubes, on définit l'énergie massique théorique transmise par les aubes,

$$
\Delta\left(U C_{u}\right)=U_{2} C_{u 2}-U_{1} C_{u 1}
$$

ainsi que l'énergie effective reçue par le fluide,

$$
\frac{\Delta P^{0}}{\rho}=\frac{P_{2}^{0}-P_{1}^{0}}{\rho}
$$

A l'entrée de la roue, la pression totale est déduite à partir de la pression statique mesurée en une section amont située assez loin du champ d'action de la roue et non affectée par la recirculation qui apparaît à sous débit. Le rendement local de chaque tube s'écrit alors :

$$
\eta_{h}=\frac{\Delta P^{0} / \rho}{\Delta U C_{u}}
$$

La figure 13 montre la distribution, à différents débits, du rendement calculé entre le plafond et la ceinture. D'une façon générale, le rendement de la roue est meilleur pour les filets situés à proximité du plafond. Ceci peut être rattaché au fait que des vitesses plus importantes du côté de la ceinture engendrent des pertes par frottement plus importantes. A noter qu'à faible débit, le calcul du rendement local n'a aucune signification dans les régions où la recirculation se manifeste.

\section{VI $\square$ CONCLUSION}

Cette étude ne peut prétendre servir à tirer des conclusions définitives sur l'influence du diffuseur et de la volute. Néanmoins, elle a permis, d'une part d'avoir une meilleure idée sur l'évolution de l'écoulement, et, d'autre part, de compléter la base de données relative à la roue SHF servant comme cas test pour la validation des codes de calcul intégrant ce genre de configuration. Les essais ont montré une forte dépendance vis-à-vis du diffuseur. L'influence de la volute semble quant à elle limitée à la région du bec de volute. Il faut donc retenir que pour ce genre de machine, les données issues d'une seule section en sortie de roue ne peuvent être représentatives de l'écoulement réel. Ces conclusions concernent essentiellement l'écoulement moyen en sortie de roue, elles restent insuffisantes pour expliquer les mécanismes conduisant à une telle structure de l'écoulement. Une poursuite de ces travaux devra consister à effectuer des essais dans des conditions analogues au moyen de techniques de mesure rapides.
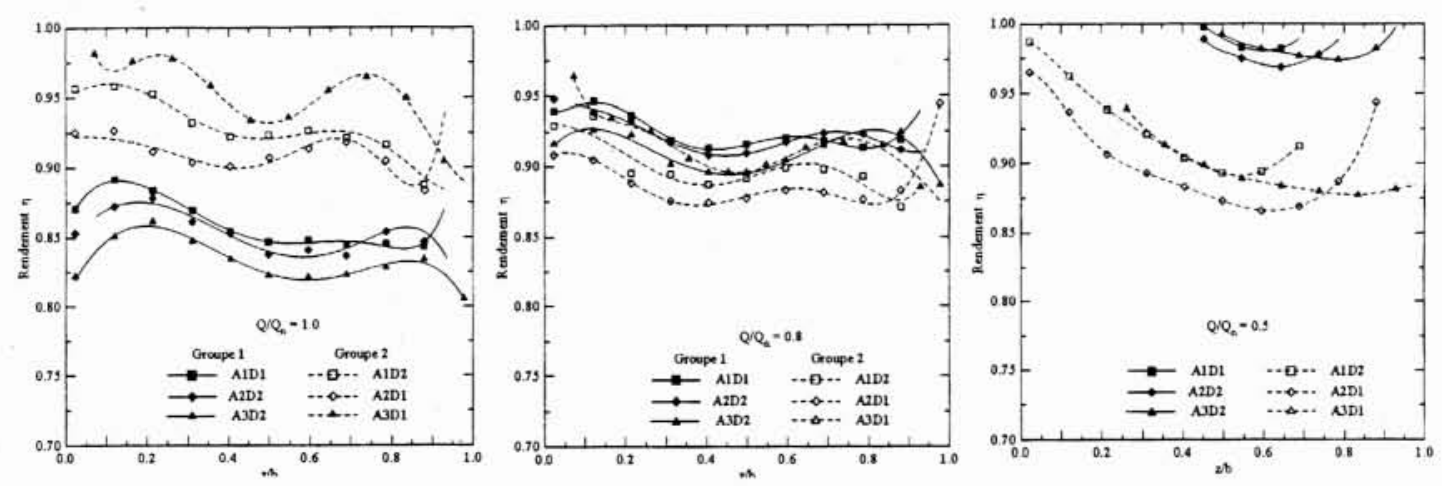

13. Rendement de la roue à $Q_{n}(\mathrm{a}), 0,8 Q_{n}(\mathrm{~b}), 0,5 Q_{n}(\mathrm{c})$. 


\section{BIBLIOGRAPHIE}

[1] Ayder E., Van Den Braembussche R., Brasz J.-J. (1993). - Experimental and Theoretical Analysis of the Flow in a Centrifugal Compressor Volute. Journal of Turbomachinery, Vol. 115, n’ 3, pp. 582-589.

[2] Barrand J.-P., Caignaert G., Graeser J.-E. et Rieutord E (1985). - Investigations expérimentales sur le canal de retour d'une turbine-pompe pour différents points de fonctionnement. La Houille Blanche, 1985, n`5, pp. 405-420.

[3] Bois G., Rieutord E. (1990). - Etude de l'écoulement en sortie de roue de pompe centrifuge depuis le débit nominal jusqu'au débit critique de recirculation. Ecully (Fr) : METRAFLU, Rapport d'étude n 88 H 0636, 169 p.

[4] Dong R., Chu S., Katz J. (1992), - Quantitative visualization of the flow within the volute of a centrifugal pump. part $B$ : results and analysis. Journal of fluids Engineering, Vol. 114, n 4, pp. 396-403.

[5] El. Hajem (1996). - Etude de l'écoulement dans une pompe centrifuge fonctionnant à débit partiel. Thèse de Doctorat, Institut National des Sciences Appliquées de Lyon. $180 \mathrm{p}$.

[6] El MARJAnl A., Bols G.. (1985). - Investigations expérimentales sur le canal de retour d'une turbine-pompe pour différents points de fonctionnement. La Houille Blanche, $1985, n^{\circ} 5$, pp. $442-454$

[7] LIU C.H., Vafidis C., Whitelaw J.H. (1994). - Flow characteristics of a centrifugal pump. Journal of fluids Engineering, Vol. 116, n², pp. 303-309.

[8] Maroufi S. (1986). - Contribution à l'étude de l'apparition des recirculations en sortie de roue dans une pompe centrifuge fonctionnant à débits partiels. Thèse de Doctorat, Université de Lille, 150 p.
[9] Miner S. M., Beaudoin R.-J., Flack R.-D. - Laser velocimeter measurements in a centrifugal flow pump. Journal of Turbomachinery, 1989, Vol. 111, n 3, pp. 205-212.

[10] MoRel. P. (1993). - Ecoulements décollés dans une pompe centrifuge. Thèse de Doctorat, Université de Lille, $210 \mathrm{p}$.

[11] SEN M. (1978). - Prerotation in Centrifugal pumps. VON KARMAN INSTITUTE FOR FLUID DYNAMICS, Lecture series 1978-3, March 6-8.

\section{NOTATION}

b largeur en sortie de roue

$C$ : vitesse de l'écoulement

$H_{n}$ : hauteur nominale de la roue

$p$ : pression statique

$p^{0}:$ pression totale : $p_{0}=p+\frac{1}{2} p C^{2}$

$Q_{n}$ : débit nominal de fonctionnement de la roue

$Q^{*}:$ débit relatif: $Q^{*}=Q / Q_{n}$

$R_{2}$ : rayon en sortie de roue

$U_{2}$ : vitesse d'entraînement en sortie de roue $U_{2}=\omega R_{2}$

$z: \quad$ position axiale du point de mesure

$\beta_{2}$ : angle de l'écoulement en sortie de roue mesuré par rapport à la direction périphérique

$\xi$ : coefficient de glissement

$\omega$ : vitesse de rotation

\section{INDICES}

$a, r, u$ : composante axiale, radiale et tangentielle de la vitesse

$c: \quad$ ceinture

$p$ : plafond

1, 2: entrée, sortie de la roue. 